\title{
Photoluminescence immunosensor based on bovine leukemia virus proteins immobilized on the $\mathrm{ZnO}$ nanorods
}

\author{
Roman Viter ${ }^{\mathrm{a}, \mathrm{f}, *}$, Maryna Savchuk $^{\mathrm{a}, \mathrm{b}, \mathrm{d}}$, Nickolay Starodub ${ }^{\mathrm{b}}$, Zigmas Balevicius ${ }^{\mathrm{c}}$, \\ Saulius Tumenas ${ }^{c}$, Almira Ramanaviciene ${ }^{\mathrm{d}}$, Daniels Jevdokimovs ${ }^{\mathrm{a}}$, Donats Erts ${ }^{\mathrm{a}}$, \\ Igor Iatsunskyi ${ }^{\mathrm{e}, *}$, Arunas Ramanavicius ${ }^{\mathrm{d}, *}$ \\ ${ }^{a}$ Institute of Chemical Physics, and Institute of Atomic Physics and Spectroscopy, University of Latvia, 19 Raina Boulevard, LV 1586 Riga, Latvia \\ ${ }^{\mathrm{b}}$ National University of Life and Environmental Sciences, 15, Geroyiv Oborony, Kyiv 03041, Ukraine \\ ${ }^{\mathrm{c}}$ State Research Institute Center for Physical Sciences and Technology, Savanoriu ave. 231, LT-01108 Vilnius, Lithuania \\ ${ }^{\mathrm{d}}$ NanoTechnas, Center of Nanotechnology and Materials Science, Faculty of Chemistry and Geosciences, Vilnius University, Naugarduko 24, LT-03225, Vilnius, Lithuania \\ ${ }^{\mathrm{e}}$ NanoBioMedical Centre, Adam Mickiewicz University, 85 Umultowska str., 61-614, Poznan, Poland \\ ${ }^{\mathrm{f}}$ Sumy State University, Medical Institute, Department of Public Health, 31, Sanatornaya st., 40018, Sumy, Ukraine
}

\section{A R T I C L E I N F O}

\section{Keywords:}

Optical immunosensor

Bovine leukemia virus (BLV)

$\mathrm{ZnO}$ nanorods

Photoluminescence

Antigen-antibody complex

\begin{abstract}
A B S T R A C T
Bovine leukaemia virus (BLV) proteins gp51, which are serving as antigens for specific antibodies against BLV proteins (anti-gp51), were applied as biological recognition part in the design of immunosensor devoted for the determination of anti-gp51. The efficiency of the immobilization of BLV proteins gp51 on $\mathrm{ZnO}$ nanorod (ZnONR) modified glass (ZnO-NR/glass) surface was evaluated. The formation of antigen-antibody complex on the $\mathrm{ZnO} /$ glass modified by the BLV proteins gp51 (gp51/ZnO-NR/glass) was investigated by the determination of changes in $\mathrm{ZnO}$ photoluminescence. The applicability of gp51/ZnO-NR/glass in the design of photoluminescence based immunosensor was evaluated. Bovine serum albumin (BSA) was applied for the modification of sensing gp51 layer in order to form gp51\&BSA layer with advanced selectivity. Polyallylamine hydrochloride (PAH) was applied in order to improve the immobilization of gp51 and BSA based sensing layer (gp51\&BSA) on the surface of ZnO-NR/glass. PAH was applied during the formation of gp51\&BSA/PAH/ZnO-NR/glass structure. Some aspects of the mechanism of interaction between biomolecules (gp51, BSA and anti-gp51) and ZnO-NR during the preparation and action of gp51\&BSA/ZnO-NR/glass- and gp51\&BSA/PAH/ZnO-NR/glass-based immunosensors have been discussed.
\end{abstract}

\section{Introduction}

Express determination of retroviral infections among humans and animals is an important global problem. Bovine leukemia virus (BLV) induces one of several recently known retroviral infections, which affects cattle all over the world and is endemic in many dairy herds [1]. Cattle of all ages may be infected by BLV but the most of them might be infected soon after the birth by ingestion of milk from an infected cow. Therefore the main way to prevent the spread of diseases caused by retroviral infection is the timely diagnosis [2]. Recently used BLV detection methods are time- and labor-consuming and therefore they do not meet recent requirements. In order to improve diagnosis a number of different BLV infection detection methods were developed, including polymerase chain reaction [3] and different types of the immunological methods such as enzyme-linked immunosorbent assay, agar gel immunodiffusion, radioimmune assay, immunoblot test $[4,5]$, etc. The traditional immunological methods have high specificity and sensitivity, but the most of them are time consuming and/or require some additional expensive materials, such as the labeled antibodies [6]. Because BLV is one of the most common infectious of cattle and is endemic in many dairy herds, thus control and eradication programs based on fast, simple, and sensitive BLV detection method are highly desirable. These requirements can be successfully solved using immunosensors [7-10].

Practical application of electrochemical immunosensors, even if they demonstrate high sensitivity to analyte and are less sensitive to the non-specific adsorption of interfering materials, still have several drawbacks: (i) sometimes additional redox mediators are required in

\footnotetext{
* Corresponding authors.

E-mail addresses: r.viter@med.sumdu.edu.ua, roman.viter@lu.lv (R. Viter), nikstarodub@yahoo.com (N. Starodub), Almira.Ramanaviciene@chf.vu.lt (A. Ramanaviciene), igoyat@amu.edu.pl (I. Iatsunskyi), Arunas.Ramanavicius@chf.vu.lt (A. Ramanavicius).
} 
order to generate amperometric response, (ii) the signal of such devices depends on characteristics of aliquot (e.g. ionic strength, $\mathrm{pH}$, etc), (iii) the regeneration of surface in order to use it for multiple analyte detection is not always successful $[6,7,11,12]$. Therefore, in some cases label-free immunosensors are more preferable. Several surface plasmon resonance (SPR) [7], optical ellipsometry [8], acoustic [9] and photoluminescence (PL) [13] based BLV sensors were developed. Most of above-mentioned sensors are optical, where metal and metal oxide based structures are applied. Some metal oxide based nanorods can provide a possibility to detect analytical signal of immunosensor directly (without the usage of additional reagents) and rapidly (within 3-5 minutes), inexpensively (cost of single analysis is less as a one US dollar) [14]. Among many metal oxides zinc oxide ( $\mathrm{ZnO})$ also has attracted interest of biosensorics related researches due to suitable semiconducting properties [15], which are based on wide and direct optical band gap ( $3.3 \mathrm{eV})$, high exciton binding energy $(\sim 60 \mathrm{meV})$ and possibility to tune a band gap in the range from 2.1 to $3.5 \mathrm{eV}$. Because of here mentioned unique properties the $\mathrm{ZnO}$ is regarded as one of the most prospective materials for photonics, optoelectronics and biosensorics [15-17]. The wurtzite-based $\mathrm{ZnO}$ nanorods (NRs) showed high photoluminescence in UV range $(370-390 \mathrm{~nm})$, which is related to excitonic emission band [18] and such NRs have been used for PL based detection of Salmonella [18]. Additionally, $\mathrm{ZnO}$ nanorods with the layer of the immobilized antibodies were successfully used for interdigitated capacitive immunosensors [19], for aptamers [20] and lipid films-based electrochemical sensors [21], and some other analytical systems.

Recent developments in optoelectronics offer LEDs with output power of $0.4-1 \mathrm{~mW}$, which are in the interest of biosensorics as low power intensity light source suitable for excitation of photoluminescence. However traditional detection part of the based biosensor consist of monochromator and photomultiplier, which are required in order to enhance the low intensity signal, what makes the cost of the detection system quite high. This drawback can be solved by the application of optical fiber based technique [22]. Recently, we have developed an fiber optic-based system based on photoluminescence measurements suitable for biomedical applications [22]. It is low cost, portable, computer driven technique. Despite of this, the optical fiber system has lower spectral resolution comparing to the traditional methods, what is significant drawback during intensity measurements [22].

In this paper we have evaluated the application of $\mathrm{ZnO}$ nanorods (ZnO-NRs) in the design of optical fiber based photoluminescence immunosensor, which is dedicated for the determination of specific antibodies produced in cattle as humoral immune response against BLVantigens that mainly consist of protein gp51.

\section{Experimental}

\subsection{Materials}

BLV proteins gp51, and two cattle serums (i) 'BLV-positive', which has specific antibodies against BLV proteins, and (ii) 'BLV-negative', which is free from specific antibodies against BLV proteins gp51, all were purchased from "Leykonad", Poltava, Ukraine. Glass slides, polyallylamine hydrochloride (PAH), butanol and bovine serum albumin (BSA) were obtained from Sigma-Aldrich (St. Louis, USA). All solutions were prepared using distilled and deionized water (Milli Q-plusMillipore system).

\subsection{Preparation and characterization of $\mathrm{ZnO}-\mathrm{NRs}$ sensitive layer}

ZnO nanorod powder was synthesized as reported previously [22]. Colloidal suspension of ZnO-NRs in butanol (concentration $1 \mathrm{mg} / \mathrm{ml}$ ) was prepared. The solution of ZnO-NRs $(20 \mu \mathrm{L})$ was deposited on the cleaned glass substrate in order to form ZnO-NRs layer on the glass surface. The samples were dried at room temperature and annealed in muffle furnace at $450{ }^{\circ} \mathrm{C}$ in air for $3 \mathrm{~h}$. Scanning electron microscopy (SEM) imaging of the prepared samples was performed by Hitachi S4800 SEM (Mannheim Office) (Japan).

Optical properties of the ZnO-based nanorods were studied by PL spectroscopy. Nitrogen laser of $\lambda=337 \mathrm{~nm}$ LGI-21 from Polyaron (Moscow, Russia) with $0.4 \mathrm{~mW}$ output power was used as an excitation source. The PL spectra were studied by optical fiber spectrometer, HR2000 + from Ocean Optics (Dunedin, USA) in the wavelength range of $360-420 \mathrm{~nm}$.

Raman spectrometer ALFA300R from Witek (Ulm, Germany) with $532 \mathrm{~nm}$ excitation laser source was used for transmittance Raman measurements. The Raman spectra of the samples were recorded in the range of $50-2000 \mathrm{~cm}^{-1}$.

\subsection{Functionalization of the $\mathrm{ZnO}-\mathrm{NRs} /$ glass by proteins and the evaluation} of immunosensor response

BLV proteins gp51, which served as an antigen (Ag) for anti-gp51 antibodies, were used in the development of biological recognition part of immunosensor. BLV proteins were immobilized on ZnO-nanorod (ZnO-NR) modified glass (ZnO-NR/glass) surface. The functionalization procedures, which were applied for modification of PL-based transducer surface are shown in Fig. 1.

The ZnO-NR/glass slides were washed with ethanol, dried and then rinsed with physiological (0.85\%) solution of $\mathrm{NaCl}$. Initial $\mathrm{BLV}$ protein gp51, which was acting as antigen (Ag) in this bioanalytical system, containing sample was diluted to the concentration of $0.5 \mathrm{mg} / \mathrm{L}$ with the solution of $\mathrm{NaCl}$ and such diluted solutions were incubated on the surface of $\mathrm{ZnO}-\mathrm{NR} /$ glass substrates for $20 \mathrm{~min}$ in order to get layered gp51/ZnO-NR/glass structure. Then gp51/ZnO-NR/glass surface was rinsed with the physiological solution of $\mathrm{NaCl}$ and dried in air at room temperature. In order to prevent non-specific adsorption of proteins, the gp51/ZnO-NR/glass slides were incubated in $0.5 \mathrm{mg} / \mathrm{mL}$ solution of BSA for $20 \mathrm{~min}$ (Fig. 1A). The PL-spectra were registered before and after each incubation step.

The deposition of polyallylamine hydrochloride $(\mathrm{PAH})$ and investigation of modified surfaces. In order to get PAH-modified ZnO-NR/glass (PAH/ $\mathrm{ZnO} /$ glass) aqueous solution of $20 \mu \mathrm{g} / \mathrm{mL}$ of $\mathrm{PAH}$ was incubated for $20 \mathrm{~min}$ on the surface of $\mathrm{ZnO}-\mathrm{NR} /$ glass substrate and then it was washed with distilled water, and dried in air at room temperature. The immobilization of gp51 and BSA on the PAH/ZnO-NR/glass was performed similarly to the procedure, which is described for ZnO-NR/glass substrate (Fig. 1B) and in such way gp51\&BSA/PAH/ZnO-NR/glassbased immunosensing structure was formed. PL-spectra were recorded before and after each modification step.

During the determination of antibodies against BLV proteins gp51 (gp51), the gp51/ZnO-NR/glass and gp51/PAH/ZnO-NR/glass samples were incubated for $20 \mathrm{~min}$ in 'BLV-positive' cattle serum containing anti-gp51 and in 'BLV-negative' cattle serum aliquot not-containing anti-gp51. Before the incubation both 'BLV-positive' and 'BLV-negative'

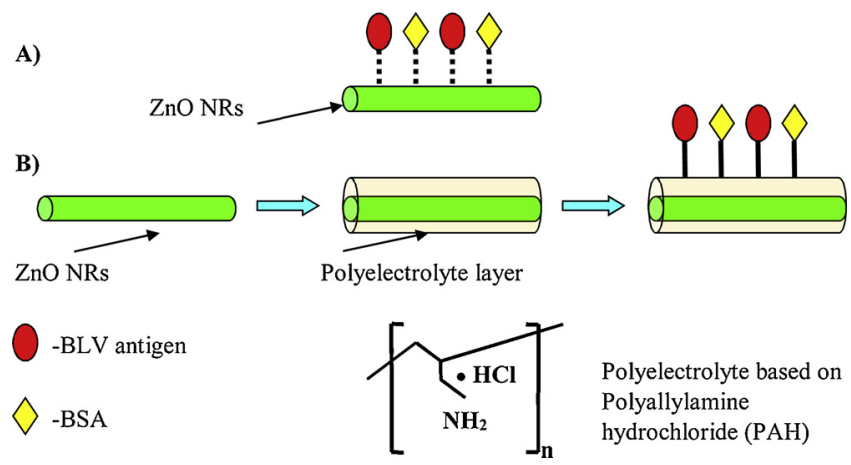

Fig. 1. Scheme of gp51 protein and BSA immobilisation on the surface of $\mathrm{ZnO}$ $\mathrm{NR} /$ glass (A) and on - PAH/ZnO-NR/glass (B). 

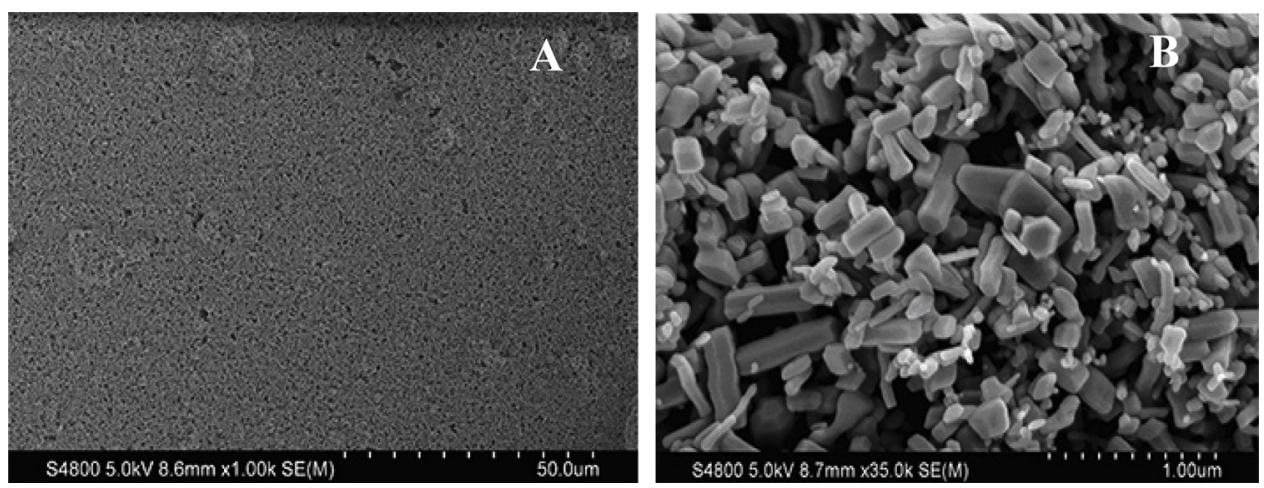

Fig. 2. SEM images of deposited $\mathrm{ZnO}$ nanorods with different magnification.

serums were diluted with physiological solution of $\mathrm{NaCl}$ in following ratios: $c_{1}=1: 1,000,000 \quad\left(10^{-6}\right.$ a.u. $), c_{2}=1: 100,000 \quad\left(10^{-5}\right.$ a.u. $)$, $c_{3}=1: 10,000\left(10^{-4}\right.$ a.u. $), c_{4}=1: 1000\left(10^{-3}\right.$ a.u. $)$ and $c_{5}=1: 100\left(10^{-2}\right.$ a.u).

After the incubation in the tested serums, the surfaces were washed and dried. The PL-spectra were recorded before and after each incubation step.

Additional control measurements have been performed by the incubation of PAH/ZnO-NR/glass, which was not functionalized by gp51 proteins, in 'BLV-positive' and only 'BLV-negative' cattle serums. The corresponding PL spectra have been recorded before and after the incubation in 'BLV-positive' and 'BLV-negative' cattle serums.

Each analytical measurement was performed at least 3 times and the average of registered signals is presented.

\section{Results and discussion}

\subsection{Characterization of $\mathrm{ZnO}$ nanorods}

A SEM image of the ZnO-NRs deposited on a glass substrate (ZnO$\mathrm{NR} /$ glass) is shown in Fig. 2A,B. From the image it is seen that the ZnOnanorods had random orientation (Fig. 2B). The distribution of the diameter of formed ZnO-NRs is $60-100 \mathrm{~nm}$ and the length is $300-900 \mathrm{~nm}$ as reported in [22].

Raman spectrum of $\mathrm{ZnO}-\mathrm{NR} /$ glass-based substrate is shown in Fig. 3. The Raman peak at $383 \mathrm{~cm}^{-1}$ correspond to $A_{1}(T O)$ polar optical phonon mode, peak at $439 \mathrm{~cm}^{-1}$, which is assigned to $E_{2}$ (high) nonpolar mode, and peak at $332 \mathrm{~cm}^{-1}$ that can be assigned to $\mathrm{E}_{2}^{\text {high }}-\mathrm{E}_{2}^{\text {low }}$ mode [23]. Raman peaks, which are observed between 570 and $650 \mathrm{~cm}^{-1}$, can be associated with structural disorders, such as oxygen vacancies, $\mathrm{Zn}$ interstitial and their combination [18]. Sharp Raman $\mathrm{E}_{2}$ mode and SEM images approve the perfect wurtzite structure of $\mathrm{ZnO}$ nanorods.

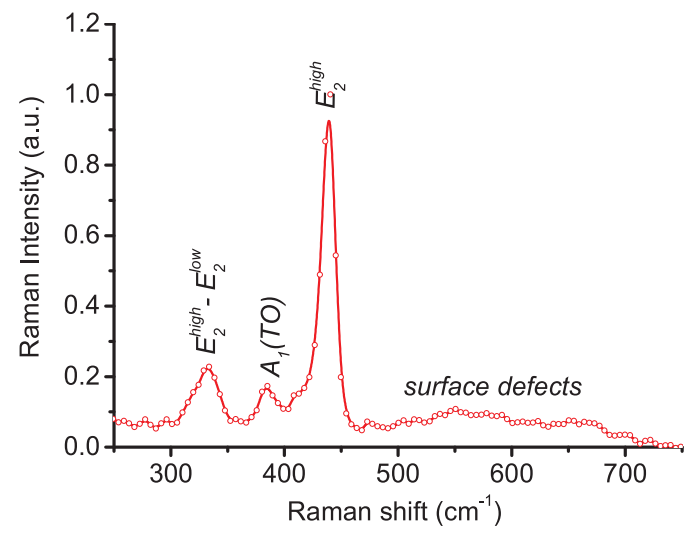

Fig. 3. Raman spectrum of ZnO-NRs deposited on glass substrate.

\subsection{Testing of optical immunosensor based on $\mathrm{ZnO}-\mathrm{NRs}$}

As it was previously reported by Viter et al. [18], UV emission band was dominant in PL-spectra of ZnO-NRs and VIS emission band was meaningless comparing to the UV band. Thus, the main attention to changes in UV emission band of ZnO-NRs was paid during the evaluation of developed immunosensor response.

After the immobilization of gp51 and blocking of gp51/ZnO-NR/ glass and gp51/PAH/ZnO-NR/glass by BSA gp51\&BSA/ZnO-NR/glass and gp51\&BSA/PAH/ZnO-NR/glass structures were formed. Both gp51 \&BSA/ZnO-NR/glass and gp51\&BSA/PAH/ZnO-NR/glass structures were analyzed by photoluminescence spectroscopy, in the same way as it was reported by Viter et al. [18] and Syshchyk et al. [24]. The registered PL-spectra are presented in Fig. 4.

PL-spectra of bare ZnO-NR/glass substrate have significantly changed after the immobilization of gp51 and BSA. Particularly, the immobilization of gp51 resulted in the decrease of the PL-intensity, whereas modification of gp51/ZnO-NR/glass by BSA resulted in the increase of the PL-intensity (Fig. 4A). The formation of PAH-polyelectrolyte layer on the ZnO-NR/glass surface (PAH/ZnO-NR/glass) resulted in the increase of the photoluminescence (Fig. 4B). The immobilization of gp51 and BSA on the PAH/ZnO-NR/glass surface resulted in the formation of gp51\&BSA/PAH/ZnO-NR/PAH structure. During the modification step of PAH/ZnO-NR/glass by gp51 increased $\mathrm{PL}$ was registered for gp51\&BSA/PAH/ZnO-NR/glass and after next modification step by BSA decrease of the PL intensity was observed for gp51\&BSA/PAH/ZnO-NR/glass structure (Fig. 4B). The differences of PL-spectra during the adsorption of gp51 and BSA proteins on the PAH modified ZnO-NR/glass- and not modified PAH/ZnO-NR/glass-based substrates is attributed to electrostatic effect of $\mathrm{PAH}$.

The formation of bioselective layers (gp51 or gp51\&BSA) is affected by electrostatic interaction between proteins (gp51 and BSA) and the ZnO-NR/glass substrate. This electrostatic interaction is influencing PLspectra of modified ZnO-NR/glass substrate, which leads to the change of surface potential of $\mathrm{ZnO}[15,18,25-27]$. It is known, that UV band of ZnO-NRs can be tailored by the variation of depleted layer width, which is controlling photo-induced charge separation in near interphase between $\mathrm{ZnO}$ and solution or air [25]. As it is reported previously in our recent reviews on metal oxide biosensors, there is still no very solid explanation for the mechanism of interaction between biomolecules and $\mathrm{ZnO}$ surface $[15,18,23,28]$. Therefore, the interaction mechanism at some extent can be assessed by the evaluation of direct and polymer assisted immobilization of biomolecules on the ZnO-NRs surface.

When the gp51 and BSA molecules are adsorbed on the surface of ZnO-NRs they can mostly interact with the surface of ZnO-NRs via electrostatic interaction and via Van-der-Waals forces [6]. Isoelectric points (IEP) of ZnO, BSA and BLV-antigens are at pH 9.3, pH 4.7 and $\mathrm{pH}$ 6.4-6.6, respectively $[18,29,30]$. Based, on the fact that the $\mathrm{pH}$ in here used water solution of $\mathrm{NaCl}$ was 6.2-6.5, we can suppose that at this $\mathrm{pH}$ BSA proteins are charged negatively and gp51 proteins are at neutrally 

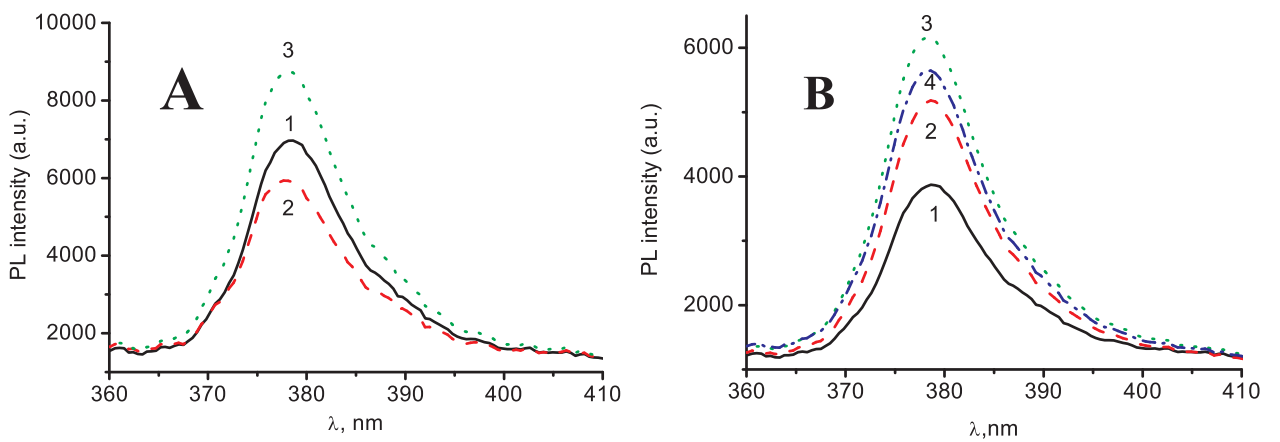

Fig. 4. PL-spectra of bare $\mathrm{ZnO}-\mathrm{NR} /$ glass substrate (A): 1 - ZnO-NRs/glass, 2 - gp51/ZnONRs/glass, 3 - gp51\&BSA/ZnO-NRs/glass; and PAH-modified ZnO nanorods (B): 1 - ZnONRs/glass, 2 - PAH/ZnO-NRs/glass, 3 - gp51/ $\mathrm{PAH} / \mathrm{ZnO}-\mathrm{NRs} /$ glass, 4 - gp51\&BSA/PAH/ ZnO-NRs/glass after the immobilization of BLV-antigen (gp51) and the blockage of remaining free binding sites with BSA. The curves are average of 3 measurements at each stage of modification. charged state, whereas ZnO-NRs surface after the adsorption of gp51 proteins is charged positively. Therefore, due to electrostatic interaction the adsorption of gp51 proteins on bare $\mathrm{ZnO}-\mathrm{NR} /$ glass substrate exhibited opposite effect to the intensity of PL-signal of ZnO-NRs in comparison to that induced by BSA proteins.

Polyelectrolyte - PAH - is also involved into variation of $\mathrm{ZnO}$ surface charge. PAH is supposed to provide better electrostatic attraction of gp51 to the ZnO-NRs surface via the polycationic layer of PAH [31-34] and the formation of polyelectrolyte layer on the $\mathrm{ZnO}$ surface resulted the increase of $\mathrm{ZnO}$ photoluminescence due to the passivation of centers, corresponding to non-radiative recombination or/and the decrease of the depletion layer in surface region of ZnO-NRs.

The surface of PAH layer has been charged positively [30]. Therefore, BLV-antigens have been bound to PAH via negatively charged groups such as $-\mathrm{COO}^{-},-\mathrm{COH},-\mathrm{OH}$, etc. The increase of PL the most probably is related to electrostatic interactions between ZnO-NRs, PAH and BLV-antigens, because gp51 are not able to transfer charge to the ZnO-NRs.

Before the investigation of bovine serum samples the response of immunosensor towards 'BLV-negative' and 'BLV-positive' (anti-gp51 antibodies containing) serum probes were tested (Fig. 5A,B). From the data obtained for ZnO-NR/glass samples, the non-specific adsorption of materials, which are present in the 'BLV-positive' probe, results in the decrease of PL-signal, whereas the non-specific adsorption of materials, which are present in the 'BLV-negative' probe, results in to the increase of the PL-signal (Fig. 5A). Non-specific interaction of 'BLV-positive' and 'BLV-negative' probes with $\mathrm{PAH} / \mathrm{ZnO}-\mathrm{NR} /$ glass surface resulted in the increase of the PL-signal intensity (Fig. 5B). Comparing the changes of ZnO-NR/glass- and PAH/ZnO-NR/glass-based immunosensor signals during the functionalization by proteins we can assume that the formation of PAH/ZnO-NR/glass structure, resulted in the change of interaction between the PL-centers of ZnO-NRs and proteins (gp51 and BSA), which are forming bioselective layer (Figs. 4 and 5) [18,24].

The interaction of 'BLV-negative' probe with the surface of bare ZnO-NR/glass structure resulted in the increase of the PL-signal. The interaction of 'BLV-negative' probe with $\mathrm{PAH} / \mathrm{ZnO}-\mathrm{NR} /$ glass structure resulted in the increase of the PL signal.

The responses of biofunctionalized gp51\&BSA/ZnO-NR/glass and gp51\&BSA/PAH/ZnO-NR/glass structures towards 'BLV-positive' and
'BLV-negative' aliquots are shown in Figs. 6 and 7, respectively. The decrease of the PL-signal was observed when both, above mentioned, gp51-modified structures were incubated in 'BLV-positive' aliquots. It is worth to mention that after specific interaction observed signal, which is based on PL quenching, is more significant than the signal, which is based on non-specific interaction of 'BLV-positive' aliquot with $\mathrm{ZnO}$ $\mathrm{NR} /$ glass and $\mathrm{PAH} / \mathrm{ZnO}-\mathrm{NR} /$ glass structures, which were not modified by gp51 that are specifically binding anti-gp51 antibodies present in 'BLV-positive' aliquot. Both gp51\&BSA/ZnO-NR/glass and gp51\&BSA/ $\mathrm{PAH}-\mathrm{ZnO}$-NR/glass structures after the incubation in 'BLV-negative' aliquots showed the increase of the PL-signal, while after the incubation in 'BLV-positive' aliquots the signals of both (gp51\&BSA/ZnO-NR/glass and gp51\&BSA/PAH-ZnO-NR/glass) structures has decreased.

The response of developed immunosensors was calculated according to the following equation, which was reported earlier $[18,35]$ :

$S=1-I_{\text {norm }}(C)$,

where $I_{\text {norm }}(C)$ is a normalized PL intensity after the treatment of gp51\& $\mathrm{BSA} / \mathrm{ZnO}-\mathrm{NR} /$ glass structure with 'BLV-positive' or 'BLV-negative' probe at corresponding probe concentration $C$.

The calibration curves of $\mathrm{ZnO}-\mathrm{NR} /$ glass and $\mathrm{PAH} / \mathrm{ZnO}-\mathrm{NR} /$ glass based structures towards 'BLV-positive' and 'BLV-negative' BLV probes are shown in Fig. $8 \mathrm{~A}$ and $\mathrm{B}$, respectively. In presented results some differences of PL-signal of these two ZnO-NR/glass and PAH/ZnO-NR/ glass based structures after interaction with 'BLV-positive' and 'BLVnegative' probes are observed.

The incubation of gp51\&BSA/ZnO-NR/glass and gp51\&BSA/PAH/ ZnO-NR/glass based structures in 'BLV-positive' aliquots resulted in the decrease of PL due to interaction of immobilized gp51 proteins with anti-BLV antibodies. Form shifts of PL-spectra maximums we can conclude that the dominating effect on photoluminescent centres of $\mathrm{ZnO}$ NRs based on electrostatic interaction between ZnO-NRs and immobilized gp51 in similar way as it was determined in the case of interaction between $\mathrm{TiO}_{2}$ and gp51 [36].

We can conclude that no significant changes between 1:1,000,000 and 1:100,000 dilution of probes were observed for gp51\&BSA/ZnONR/glass based immunosensor (Fig. 8A), whereas for immunosensor, which was based on gp51\&BSA/PAH ZnO-NR/glass, the PL-signal at the
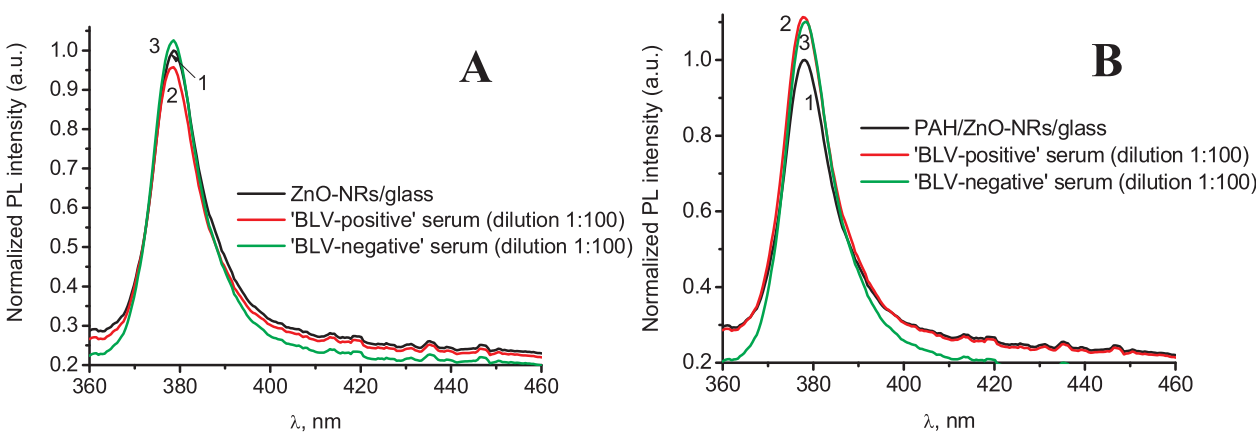

Fig. 5. Control measurements of 'BLV-positive' and 'BLV-negative' serum probes on ZnO-NR/ glass (A): 1 - ZnO-NRs/glass, 2 - BLV-positive' probe/ZnO-NRs/glass, 3 - BLV-negative' probe/ZnO-NRs/glass and PAN/ZnO-NR/glass (B): 1 - PAH/ZnO-NRs/glass, 2 - BLV-positive' probe/PAH/ZnO-NRs/glass, 3 - BLV-negative' probe/PAH/ZnO-NRs/glass. The curves are average of 3 measurements at each stage of modification. 

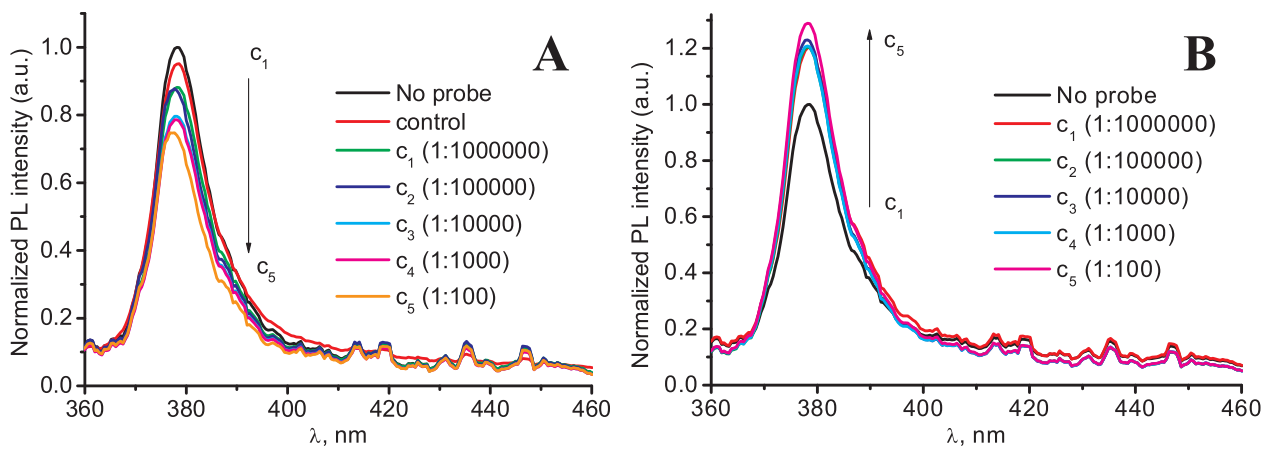

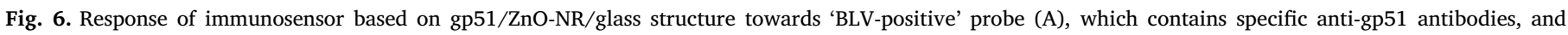

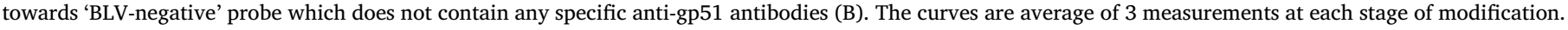
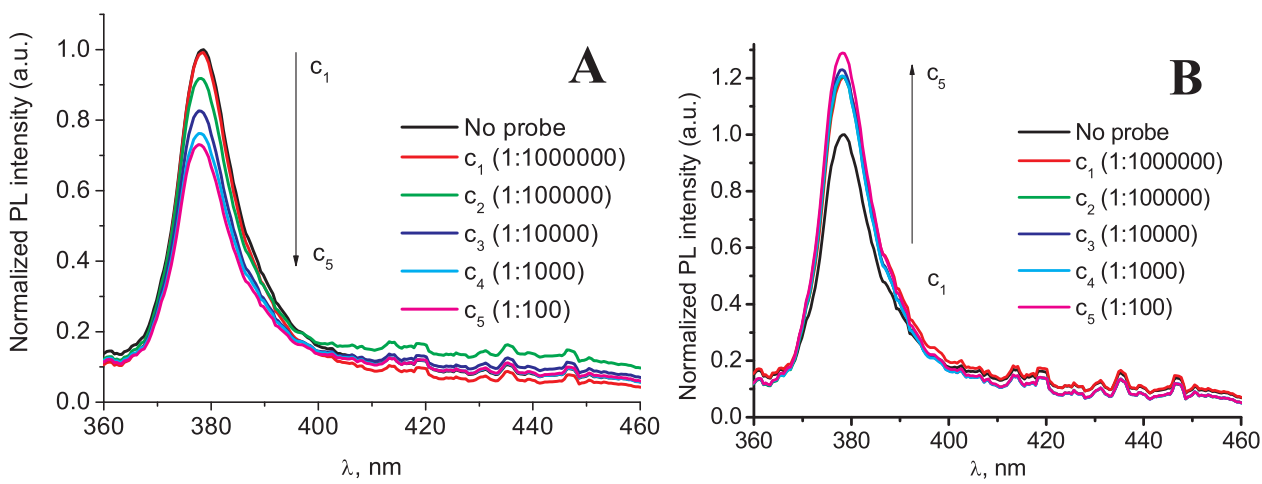

Fig. 7. Response of immunosensor based on gp51\&BSA/PAH/ZnO-NR/glass structure towards 'BLV-positive' probe (A), which contains specific anti-gp51 antibodies, and towards 'BLV-negative' probe which does not contain any specific anti-gp51 antibodies (B). The curves are average of 3 measurements at each stage of modification.

same concentration level has increased up to $10 \%$.

It is worth to mention, that the direct and polyelectrolyte - PAH assisted immobilization of BLV-antigens on the ZnO-NR/glass surface provided to gp51\&BSA/ZnO-NR/glass-based immunosensor advanced sensitivity against BLV antibodies [18]. However, random orientation of BLV-antigens significantly reduced the sensitivity of gp51\&BSA/ZnONR/glass-based immunosensor towards anti-gp51 antibodies and analytical signal saturation was observed even at the dilution of 'BLV-positive' serum at 1:10,000. Contrary to this, gp51\&BSA/PAH/ZnO-NR/ glass-based immunosensor showed good linearity of signal in the serum dilution range of 1:1,000,000 - 1:1000 of 'BLV-positive'. The developed gp51\&BSA/PAH/ZnO-NR/glass-based immunosensor can well differentiate 'BLV-positive', and 'BLV-negative' serums in relatively high dilution range from 1:1000 to $1: 100,000$. Selectivity tests of gp51\&BSA/ ZnO-NR/glass- and gp51\&BSA/PAH/ZnO-NR/glass-based sensors are represented supplementary material figure $\mathrm{S} 2$.

\section{Conclusion}

Novel photoluminescence system based on gp51\&BSA/ZnO-NR/ glass- and gp51\&BSA/PAH/ZnO-NR/glass-based immunosensor for the express detection of specific antibodies against BLV protein gp51 has been reported. The system is able to discriminate between 'BLV-positive' and 'BLV-negative' blood serum probes in the dilution range from 1:1000 to 1:100,000. The proposed mechanism of PL-signal generation is mostly based on electrostatic interaction between immobilized/interacting biomolecules and $\mathrm{ZnO}-\mathrm{NRs}$.

\section{Acknowledgements}

The conducted research has been partially supported by project H2020-MSCA-RISE-2017, 'Novel 1D photonic metal oxide nanostructures for early stage cancer detection' (Project number: 778157); Ukraine-Latvia bilateral project 'Algoritma optimizēšana audzēju progresēšanas un ārstēšanas efektivitātes biokịmisko raksturlielumu noteikšanai ar biosensoriem' (LV-UA/2016/6, contract nr. ZD2016/ 20322). I.I. acknowledges the financial support from the National Science Centre of Poland by the SONATA 11 project UMO-2016/21/D/ ST3/00962. A.R. and A.R. are grateful to Ukrainian-Lithuanian bilateral research project "Application of hybrid nanostructures which are based
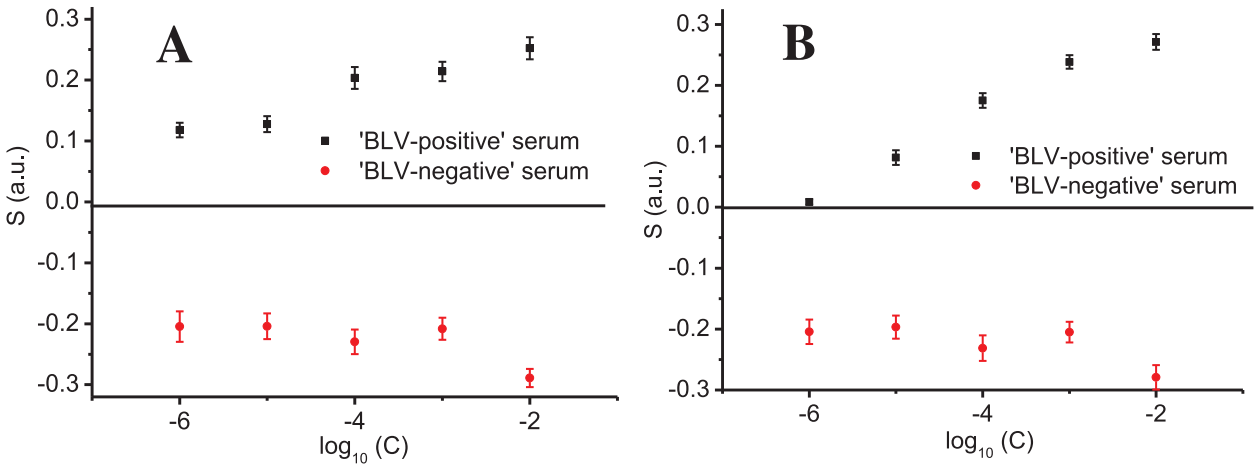

Fig. 8. Calibration plots of immunosensor signal $\mathrm{S}$ based on gp51\&BSA/ZnO-NR/glass (A) and gp51\&BSA/PAH/ZnO-NR/glass (B) modified surfaces towards differently diluted 'BLVnegative' and 'BLV-positive' serums with dilution $C$ (The plots are shown in semi logarithmic scale). Here $S=1-I_{\text {norm }}(C)$ (according to Eq. (1)), where $I_{\text {norm }}(C)$ is a normalized PL intensity after the treatment of gp51\&BSA/ZnONR/glass structure with 'BLV-positive' or 'BLVnegative' probe at corresponding probe concentration $C$; $\log _{10}(C)$ - is a logarithm of concentration, taking into account that the concentration of initial (undiluted sample) is equal to 1 . 
on $\mathrm{TiO}_{2}$ or $\mathrm{ZnO}$ and modified by biomolecules, in optoelectronic sensors" Lithuanian Research Council project No P-LU-18-53.

\section{Appendix A. Supplementary data}

Supplementary material related to this article can be found, in the online version, at doi:10.1016/j.snb.2019.01.054.

\section{References}

[1] B. Kurtinaitiene, D. Ambrozaite, V. Laurinavicius, A. Ramanaviciene, A. Ramanavicius, Amperometric immunosensor for diagnosis of BLV infection, Biosens. Bioelectron. 23 (2008) 1547-1554.

[2] B.J.R.P.D. Kirkland, Enzootic bovine leukosis, Aust. New Zeal. Stand. Diagnostic Proced. (2005) 1-14.

[3] M. Hassanpour, M. Nadalian, S. Safi, R. Madani, S. Bokaie, Epidemiological survey of Bovine Leukemia Virus (BLV) infection and its effective factors emphasis ELISA and nested PCR in dairy herds around Babol city (North of Iran) as a Caspian climate, Eur. J. Zool. Res. 3 (2014) 166-171.

[4] D. Balić, I. Lojkić, M. Periškić, T. Bedeković, A. Jungić, N. Lemo, B. Roić, Ž. Čač, L. Barbić, J. Madić, Identification of a new genotype of bovine leukemia virus, Arch. Virol. 157 (2012) 1281-1290.

[5] B. Choudhury, C. Finnegan, J.P. Frossard, C. Venables, F. Steinbach, Colostrum replacer and bovine leukemia virus seropositivity in calves, Emerg. Infect. Dis. 19 (2013) 1027-1028.

[6] I. Schwartz, D. Levy, Pathobiology of bovine leukemia virus, Vet. Res. 25 (1994) 521-536.

[7] A. Ramanaviciene, N. German, A. Kausaite-Minkstimiene, J. Voronovic, J. Kirlyte, A. Ramanavicius, Comparative study of surface plasmon resonance, electrochemical and electroassisted chemiluminescence methods based immunosensor for the determination of antibodies against human growth hormone, Biosens. Bioelectron. 36 (2012) 48-55.

[8] J. Baniukevic, J. Kirlyte, A. Ramanavicius, A. Ramanaviciene, Application of oriented and random antibody immobilization methods in immunosensor design, Sens. Actuators B Chem. 189 (2013) 217-223.

[9] Z. Balevicius, A. Ramanaviciene, I. Baleviciute, A. Makaraviciute, L. Mikoliunaite, A. Ramanavicius, Evaluation of intact- and fragmented-antibody based immunosensors by total internal reflection ellipsometry, Sens. Actuators B Chem. 160 (2011) 555-562.

[10] A. Makaraviciute, T. Ruzgas, A. Ramanavicius, A. Ramanaviciene, Antibody fragment immobilization on planar gold and gold nanoparticle modified quartz crystal microbalance with dissipation sensor surfaces for immunosensor applications, Anal. Methods 6 (2014) 2134-2140.

[11] A. Ramanavicius, A. Finkelsteinas, H. Cesiulis, A. Ramanaviciene, Electrochemical impedance spectroscopy of polypyrrole based electrochemical immunosensor, Bioelectrochemistry 79 (2010) 11-16.

[12] A. Ramanavicius, Y. Oztekin, A. Ramanaviciene, Electrochemical formation of polypyrrole-based layer for immunosensor design, Sens. Actuators B Chem. 197 (2014) 237-243.

[13] R. Viter, N. Starodub, V. Smyntyna, A. Tereschenko, A. Kusevitch, J. Sitnik, J. Buk, J. Macakd, Immune biosensor based on silica nanotube hydrogels for rapid biochemical diagnostics of bovine retroviral Leukemia, Procedia Eng. 25 (2011) 948-951.

[14] F.S. Ligler, Perspective on optical biosensors and integrated sensor systems, Anal. Chem. 81 (2009) 519-526.

[15] A. Tereshchenko, M. Bechelany, R. Viter, V. Khranovskyy, V. Smyntyna, N. Starodub, R. Yakimova, Optical biosensors based on ZnO nanostructures: Advantages and perspectives. A review, Sens. Actuators B Chem. 229 (2016) 664-677.

[16] D. Majchrowicz, M. Hirsch, P. Wierzba, M. Bechelany, R. Viter, M. Jędrzejewska-Szczerska, Application of Thin ZnO ALD Layers in Fiber-Optic Fabry-Pérot Sensing Interferometers, Sensors 16 (2016) 416.

[17] K. Shavanova, Y. Bakakina, I. Burkova, I. Shtepliuk, R. Viter, A. Ubelis, V. Beni,
N. Starodub, R. Yakimova, V. Khranovskyy, Application of 2D non-graphene materials and 2D oxide nanostructures for biosensing technology, Sensors 16 (2016) 223.

[18] R. Viter, V. Khranovskyy, N. Starodub, Application of Room Temperature Photoluminescence From ZnO Nano-rods for Salmonella Detection, IEEE Sens. J. 14 (2014) 2028-2034.

[19] P. Sanguino, T. Monteiro, S.R. Bhattacharyya, C.J. Dias, R. Igreja, R. Franco, ZnO nanorods as immobilization layers for interdigitated capacitive immunosensors, Sens. Actuators B Chem. 204 (2014) 211-217.

[20] Y. Nam, J. Park, Y.K. Pak, J.J. Pak, Aptamer-Based Immunosensor on the ZnO Nanorods Networks, J. Nanosci. Nanotechnol. 12 (2012) 5547-5551.

[21] I.Z. H, M. Nikolaos, N. Georgia-Paraskevi, N.D. P, W. Magnus, P. Nikolas, The development of highly sensitive and selective immunosensor based on antibody immobilized ZnO nanorods for the detection of D-Dimer, Electroanalysis 26 (2014) 292-298.

[22] R. Viter, K. Jekabsons, Z. Kalnina, N. Poletaev, S.H. Hsu, U. Riekstina, Bioanalytical system for detection of cancer cells with photoluminescent $\mathrm{ZnO}$ nanorods, Nanotechnology. 27 (2016) 465101.

[23] R. Viter, M. Savchuk, I. Iatsunskyi, Z. Pietralik, N. Starodub, N. Shpyrka, A. Ramanaviciene, A. Ramanavicius, Analytical, thermodynamical and kinetic characteristics of photoluminescence immunosensor for the determination of Ochratoxin A, Biosens. Bioelectron. 99 (2018) 237-243.

[24] O. Syshchyk, V.A. Skryshevsky, O.O. Soldatkin, A.P. Soldatkin, Enzyme biosensor systems based on porous silicon photoluminescence for detection of glucose, urea and heavy metals, Biosens. Bioelectron. 66 (2015) 89-94.

[25] A.A. Chaaya, R. Viter, M. Bechelany, Z. Alute, D. Erts, A. Zalesskaya, K. Kovalevskis, V. Rouessac, V. Smyntyna, P. Miele, Evolution of microstructure and related optical properties of ZnO grown by atomic layer deposition, Beilstein J. Nanotechnol. 4 (2013) 690-698.

[26] R. Viter, Z. Balevicius, A. Abou Chaaya, I. Baleviciute, S. Tumenas, L. Mikoliunaite, A. Ramanavicius, Z. Gertnere, A. Zalesska, V. Vataman, V. Smyntyna, D. Erts, P. Miele, M. Bechelany, The influence of localized plasmons on the optical properties of Au/ZnO nanostructures, J. Mater. Chem. C 3 (2015) 6815-6821.

[27] R. Viter, I. Iatsunskyi, V. Fedorenko, S. Tumenas, Z. Balevicius, A. Ramanavicius, S. Balme, M. Kempin, G. Nowaczyk, S. Jurga, M. Bechelany, Enhancement of Electronic and optical properties of $\mathrm{ZnO} / \mathrm{Al} 2 \mathrm{O} 3$ nanolaminate coated electrospun nano fibers, J. Phys. Chem. C. 120 (2016) 5124-5132.

[28] N.F. Starodub, K.E. Shavanova, N.F. Shpyrka, M.M. Mel'nichenko, R.V. Viter, Nanostructured materials as biosensor transducers: achievements and future developments, in: adv, Surf. Eng. Mater. (2016) 439-494.

[29] S. Ge, K. Kojio, A. Takahara, T. Kajiyama, Bovine serum albumin adsorption onto immobilized organotrichlorosilane surface: Influence of the phase separation on protein adsorption patterns, J. Biomater. Sci. Polym. Ed. 9 (1998) 131-150.

[30] H.C. McDonald, D.C. Graves, J.F. Ferrer, Isolation and characterization of an antigen of the bovine C-type virus, Cancer Res. 36 (1975) 1251-1257.

[31] A.V. Nabok, A. Tsargorodskaya, A.K. Hassan, N.F. Starodub, Total internal reflection ellipsometry and SPR detection of low molecular weight environmental toxins, Appl. Surf. Sci. 246 (2005) 381-386.

[32] D. Nikolelis, T. Varzakas, A. Erdem, G.-P. Nikoleli, Portable Biosensing of Food Toxicants and Environmental Pollutants, Portable Biosens. Food Toxicants Environ. Pollut. 5247 (2013) 515-560.

[33] N.F. Starodub, Y.O. Ogorodniichuk, O.O. Novgorodova, D.P. Nikolelis, G. P. Nikoleli (Eds.), Efficiency of Instrumental Analytical Approaches at the Control of Bacterial Infections in Water, Foods and Feeds BT - Biosensors for Security and Bioterrorism Applications, Springer International Publishing, Cham, 2016, pp. 199-229.

[34] D.C. Kim, D.J. Kang, Molecular recognition and specific interactions for biosensing applications, Sensors. 8 (2008) 6605-6641.

[35] V. Myndrul, R. Viter, M. Savchuk, N. Shpyrka, D. Erts, D. Jevdokimovs, V. Silamikelis, V. Smyntyna, A. Ramanavicius, I. Iatsunskyi, Porous silicon based photoluminescence immunosensor for rapid and highly-sensitive detection of Ochratoxin A, Biosens. Bioelectron. 102 (2018) 661-667.

[36] A. Tereshchenko, V. Smyntyna, A. Ramanavicius, Interaction mechanism between $\mathrm{TiO}_{2}$ nanostructures and bovine leukemia virus proteins in photoluminescencebased immunosensors, RSC Adv. 8 (2018) 37740-37748. 